\title{
Modulation of specific inhibitory networks in fatigued locomotor muscles of healthy males
}

\author{
Stuart Goodall ${ }^{1}$. Glyn Howatson ${ }^{1,2} \cdot$ Kevin Thomas $^{1}$
}

Received: 22 August 2017 / Accepted: 1 December 2017 / Published online: 6 December 2017

(c) The Author(s) 2017. This article is an open access publication

\begin{abstract}
Reduced maximal force capability of skeletal muscle, as a consequence of exercise, can be due to peripheral or central fatigue mechanisms. In upper-limb muscles, neuromuscular fatigue is concurrent with reduced corticospinal excitability and increased inhibition (lengthened corticospinal silent period [CSP]; reduced short-interval intracortical inhibition [SICI] ratio). However, it is unclear whether these adjustments occur in response to fatiguing exercise of locomotor muscles. This study examined the effect of fatiguing, maximal, knee-extensor exercise on motor cortical excitability and inhibition. Thirteen males performed three 30-s maximal, isometric contractions with the dominant knee-extensors (MVC1, MVC2 and MVC3), separated by $60 \mathrm{~s}$. At the end of, and between each MVC, neuromuscular fatigue, corticospinal excitability, CSP and SICI were assessed with supramaximal stimulation of the femoral nerve, and motor cortical stimulation, respectively. Repeated MVCs caused progressive reductions in MVC $(-10,-24$ and $-29 \%$, respectively, $P \leq 0.01)$, along with significant peripheral (reductions in potentiated twitch of $-23,-53$ and $-60 \%$, respectively, $P<0.001$ ) and central (reductions in VA of $-10 \%$ and $-13 \%$ post-MVC2 and 3 , respectively, $P \leq 0.01$ ) fatigue. Following MVC1 corticospinal excitability was reduced, and remained depressed thereafter. CSP increased in duration and remained longer throughout the protocol; whereas, no change in SICI was observed. Repeated, sustained, maximal contractions of the knee-extensors elicited substantial peripheral and central fatigue that was accompanied by a concomitant reduction in corticospinal excitability. However, divergent responses exist between inhibitory networks within the motor cortex, the activity of inhibitory networks mediated by $\mathrm{GABA}_{\mathrm{B}}$ are increased, whereas those mediated by $\mathrm{GABA}_{\mathrm{A}}$ are not.
\end{abstract}

Keywords Brain $\cdot$ Contraction $\cdot$ Maximal $\cdot$ Muscle $\cdot$ Sustained

\section{Introduction}

Fatigue is a universal phenomenon characterised by sensations of tiredness and weakness during or following exertion, which is underpinned and/or modulated by multiple physiological and psychological processes. Exercise, and

Electronic supplementary material The online version of this article (https://doi.org/10.1007/s00221-017-5142-x) contains supplementary material, which is available to authorized users.

Stuart Goodall

stuart.goodall@northumbria.ac.uk

1 Department of Sport, Exercise, and Rehabilitation, Faculty of Health and Life Sciences, Northumbria University, Newcastle-upon-Tyne NE1 8ST, UK

2 Water Research Group, School of Environmental Sciences and Development, Northwest University, Potchefstroom, South Africa the consequent disruption to homeostasis, is a particularly potent stimulus to elicit fatigue, the mediators of which will vary depending on the exercise task. For exhaustive exercise of a single muscle group, fatigue is accompanied with a reduction in voluntary force (termed "muscle fatigue"; Gandevia 2001) that is primarily underpinned by adjustments in central nervous system (CNS) and muscle function. Peripheral mechanisms of fatigue refer to adjustments in contractile function, whereas central mechanisms of fatigue refer to an inability to voluntarily activate the involved muscles. Peripheral and central mechanisms of fatigue, and their contributions to the force loss experienced after exhaustive single limb exercise, have been well-studied (Carroll et al. 2017). More recently the CNS response to exhaustive exercise, in particular the excitability of motor cortical networks, have been increasingly studied (Tergau et al. 2000; Benwell et al. 2006; Maruyama et al. 2006; Takahashi et al. 2009; Hunter 
et al. 2016), but how these networks respond to lower-limb muscle fatigue is unknown.

Excitatory and inhibitory responses within the motor cortex can be studied using transcranial magnetic stimulation (TMS). When TMS is delivered over the motor cortex during a voluntary contraction, a motor evoked potential (MEP) is generated in the target muscle. As noted by many others, MEPs evoked by a standard stimulus in a resting muscle reduce following a fatiguing contraction (Brasil-Neto et al. 1993; Samii et al. 1996; Maruyama et al. 2006). When such a response in the MEP is observed, and responses to peripheral nerve stimulation are unchanged, it can be concluded that corticospinal excitability is reduced (Kotan et al. 2015). Spinal excitability is known to reduce with fatigue (McNeil et al. 2009) and this has been considered as a mechanism involved in the reduced MEP amplitude. Other possible explanations for this reduction are the change in postsynaptic properties of cortical neurons, neurotransmitter depletion (Brasil-Neto et al. 1993, 1994), or modulation of ongoing synaptic inhibition or facilitation (Taylor and Gandevia 2001; Lentz and Nielsen 2002). As fatigue develops and cortical stimulations are delivered during contraction, MEPs increase, demonstrating an increased excitability of motor cortical cells, a finding that is well established in the upper-limb (Taylor et al. 1996; Benwell et al. 2006; Yoon et al. 2012) and more recently the knee-extensors (Kennedy et al. 2016; Vernillo et al. 2017). Following the MEP evoked during voluntary contraction, there is a period of silence in electromyographic activity (EMG) termed the corticospinal silent period (CSP). The CSP shows an interruption in volitional drive from the cortex and withdrawal of descending input to the spinal motorneuron pool (Chen et al. 1999; Williams et al. 2014). In a fresh state, CSP duration has been attributed to periods of spinal refractoriness and cortical inhibition (up to $200 \mathrm{~ms}$; Chen et al. 1999; Rothwell 2009). For many years it has been thought that spinal mechanisms contribute to the initial $(50-80 \mathrm{~ms})$ part of the CSP (Fuhr et al. 1991; Ziemann et al. 1993). However, more recent evidence suggests that the CSP has a much longer, and more influential spinal component than previously thought (Yacyshyn et al. 2016). During fatigue there is a lengthening of CSP duration that is reflective of greater motor cortical inhibition (McKay et al. 1996; Taylor et al. 1996; Kennedy et al. 2016; Vernillo et al. 2017) mediated by receptor $\mathrm{B}$ of the neurotransmitter gamma-aminobutyric acid (GABA ${ }_{B}$; McDonnell et al. 2006).

Paired-pulse TMS protocols provide a strategy to directly evaluate the excitability of intracortical inhibitory and facilitatory networks within the motor cortex (Ni and Chen 2011). A sub-threshold TMS stimulus, which activates intracortical inhibitory circuits, reduces the size of an MEP elicited 2-5 ms later; a response termed short-interval intracortical inhibition (SICI; Kujirai et al. 1993). There is good evidence to show that this form of inhibition is caused by the activation of $\mathrm{GABA}_{\mathrm{A}}$ within the primary motor cortex (Ziemann et al. 1996; Di Lazzaro et al. 2000). When SICI is measured following fatiguing exercise of the upper-limb, inhibition is reduced (Benwell et al. 2006; Maruyama et al. 2006; Takahashi et al. 2011; Hunter et al. 2016) or remains unchanged (Tergau et al. 2000). Despite the importance of knee-extensor muscles to locomotion, little is known about the response of motor cortical inhibitory networks following fatiguing lower-limb exercise. No changes in SICI have been found following heavy resistance (Thomas et al. 2017b) or intermittent (Brownstein et al. 2017; Thomas et al. 2017a) exercise. Moreover, Takahashi et al. (2011) studied the effect of fatiguing, submaximal exercise, and found this to progressively reduce corticospinal excitability and SICI when measured at rest in the knee-extensors. However, recent evidence has suggested that excitability of intracortical networks should be assessed in an active, rather than resting muscle (Gruet et al. 2013; Thomas et al. 2016a), and the response in such networks following maximal fatiguing exercise of the knee-extensors, remains to be elucidated.

It is common for investigations studying fatigue to report changes in inhibition without delineating the specific alterations in networks governed by the activity of $\mathrm{GABA}_{\mathrm{A}}$ and $\mathrm{GABA}_{\mathrm{B}}$. Accordingly, the aim of the present study was to investigate the effect of repeated, maximal, isometric, kneeextensor contractions on the development of fatigue, corticospinal excitability, and inhibition. It was hypothesised that the sustained, maximal contractions would elicit a substantial level of fatigue, with a concomitant reduction in corticospinal excitability and increased inhibition.

\section{Methods}

\section{Participants}

Thirteen, recreationally active males (age, $23 \pm 2$ year; stature, $1.81 \pm 0.04 \mathrm{~m}$; body mass, $85.2 \pm 12.5 \mathrm{~kg}$ ) volunteered to participate. Participants arrived at the laboratory in a rested state, having avoided strenuous exercise in the preceding $48 \mathrm{~h}$. Volunteers also refrained from caffeine for $12 \mathrm{~h}$ and alcohol for $24 \mathrm{~h}$ prior to each trial. Prior to any experimental procedures, written informed consent was obtained from all participants and the study conformed to the latest revision of the Declaration of Helsinki. Northumbria University's Research Ethics Committee approved all procedures.

\section{Experimental design}

Participants visited the laboratory on two occasions, firstly for habituation to the measurement tools and procedures, then, secondly for the experimental trial. During the 
experimental visit participants performed three maximal, isometric voluntary contractions (MVC1, MVC2, MVC3) with the dominant knee-extensors, each sustained for $30 \mathrm{~s}$ and separated by $60 \mathrm{~s}$. Following each contraction neuromuscular function, corticospinal excitability, CSP and SICI were assessed. Each visit was separated by at least 3 days; a schematic of the experimental procedure is provided in Fig. 1.

\section{Force and EMG recordings}

During all voluntary and evoked contractions, kneeextensor force $(\mathrm{N})$ was measured using a calibrated load cell (MuscleLab force sensor 300, Ergotest Technology, Norway). The load cell was fixed to a custom-built chair and connected to a non-compliant cuff attached around the participant's dominant leg, superior to the malleoli. Participants sat upright in the chair with their hips and knees at $90^{\circ}$ of flexion, they were instructed to grasp the handles on the side of the chair for support during contractions. EMG activity was recorded from the rectus femoris $(R F)$, vastus lateralis $(V L)$ and vastus medialis $(V M)$. Surface $\mathrm{Ag} /$ $\mathrm{AgCl}$ electrodes (Kendall H87PG/F, Covidien, Mansfield, MA, USA) were placed $2 \mathrm{~cm}$ apart over the muscle bellies and a reference electrode was placed over the patella. Electrode placement was marked with permanent ink to ensure a consistent placement between both sessions. The electrodes were used to record the compound muscle action potential (M-wave) elicited by electrical stimulation of the femoral nerve and MEPs elicited by TMS. The root mean square EMG (rmsEMG) activity was measured during the first and last $5 \mathrm{~s}$ of each sustained MVC and the pre-stimulus rmsEMG ( $80 \mathrm{~ms})$ activity was measured during all 10\% MVC contractions. All rmsEMG values were normalised to the maximal $\mathrm{M}$-wave at the respective time point $\left(\mathrm{rmsEMG} / M_{\max }\right)$. Signals were amplified (gain $\times 1000$ for EMG; $\times 300$ for force, CED 1902, Cambridge Electronic Design, UK), band-pass filtered (EMG only: 20-2000 Hz), digitised (4 kHz; CED 1401, Cambridge Electronic Design, UK), acquired and analysed off line (Spike2 v7.12, Cambridge Electronic Design, UK).

\section{Femoral nerve stimulation}

Single, electrical stimuli (200 $\mu$ s pulse width) were delivered to the femoral nerve through surface electrodes (CF3200, Nidd Valley Medical Ltd, North Yorkshire, UK) using a constant-current stimulator (DS7AH, Digitimer Ltd, Welwyn Garden City, Hertfordshire, UK). In line with previous investigations from our laboratory (Goodall et al. 2015; Thomas et al. 2016b, 2017b), the cathode was positioned over the nerve, high in the femoral triangle, whilst the anode was placed midway between the greater trochanter and the iliac crest. Single stimuli were delivered to the relaxed muscle beginning at $40 \mathrm{~mA}$, the intensity was increased by $20 \mathrm{~mA}$ until a plateau occurred in twitch amplitude and M-wave $\left(M_{\max }\right)$. Supramaximal stimulation was delivered by increasing the final stimulator output by $30 \%$ (mean current, $208 \pm 57 \mathrm{~mA}$ ). The positions of the stimulating electrodes were marked with indelible ink to ensure consistent placement during each session. At each time point muscle contractility was assessed for the peripherally derived resting twitches as twitch amplitude $\left(Q_{\mathrm{tw}, \mathrm{pot}}:\right.$ maximum twitch tension) and membrane excitability was inferred from the peak-to-peak amplitude of the electrically evoked $M_{\max }$.

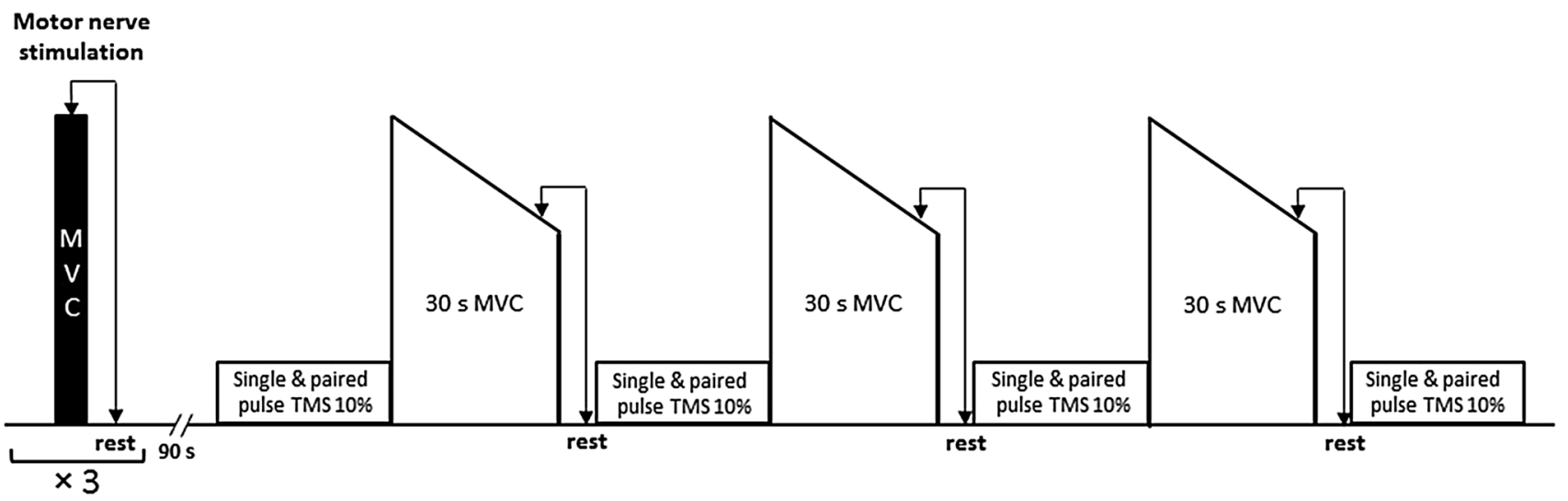

Fig. 1 Schematic of the experimental trial. At baseline participants performed 3 knee-extensor maximum voluntary contractions (MVCs) with motor nerve stimulation delivered to the femoral nerve during and $2 \mathrm{~s}$ post, to determine voluntary activation and potentiated twitch force, respectively. Single and paired responses (6 of each) to motor cortical stimulation were then elicited during a $10 \%$ MVC. Thereaf- ter, participants performed a sustained (30 s) maximal contraction with femoral nerve stimulation delivered during the final $5 \mathrm{~s}$. An additional, stimulation was delivered at rest immediately after the $30 \mathrm{~s}$ contraction, participants then contracted at $10 \%$ of the final preceding force for 1 min whilst single and paired ( 6 of each) motor cortical stimuli were delivered. The procedure was repeated 3 times 


\section{Transcranial magnetic stimulation}

Single- and paired-pulse TMS were delivered using a concave double cone coil (110 mm diameter; maximum output $1.4 \mathrm{~T}$ ), powered by a BiStim unit and two Magstim $200^{2}$ stimulators (The Magstim Company Ltd, Whitland, UK). The coil was held over the vertex to stimulate the contralateral hemisphere to the dominant leg (induced current $=$ postero-anterior). The optimal position to elicit a large MEP in the knee-extensors was identified during a brief 50\% MVC contraction, with the stimulator output set at $50 \%$. The optimal position was marked on the scalp with indelible ink to ensure a reproducible site of stimulation. Active motor threshold (aMT) was determined during a 10\% MVC as the minimum stimulus intensity that elicited a consistent MEP in the $R F$, of at least $200 \mu \mathrm{V}$ in three of five stimulations (mean stimulator output, $44 \pm 5 \%$ ).

\section{Assessment of neuromuscular function}

At baseline, MVC force was determined from three maximal 3 s contractions. Femoral nerve stimulation was delivered during each of these contractions and an additional stimulus was delivered at rest, $\sim 2 \mathrm{~s}$ after, to determine voluntary activation (VA; Merton 1954) and the $Q_{\mathrm{tw}, \mathrm{pot}}$, respectively. During the 30 -s sustained contractions, MVC was taken as the maximal force achieved in the first $5 \mathrm{~s}$ and femoral nerve stimulation was delivered during the final $5 \mathrm{~s}$. At the end of each MVC, participants were instructed to completely relax and an additional femoral nerve stimulus was delivered to determine $Q_{\mathrm{tw}, \text { pot }}$ and subsequently, VA. Following the resting stimulus, participants were instructed to contract at $10 \%$ of the preceding final MVC force for the assessment of corticospinal excitability, CSP, and SICI (see below). Once the 60-s assessment period had elapsed, participants began the next sustained maximal contraction and this procedure was repeated until 3 sustained MVCs had been completed. Strong verbal encouragement was provided throughout each sustained contraction.

\section{Corticospinal excitability, silent period, and short-interval intracortical inhibition}

During each neuromuscular assessment, six single and six paired-pulse magnetic stimuli were delivered in an alternate order, the mean responses were used to quantify corticospinal excitability and inhibition, respectively. Stimuli were separated by $4-6 \mathrm{~s}$ and delivered whilst participants held an isometric knee-extensor contraction at $10 \%$ of the final MVC force. Following each unconditioned MEP, the duration of the CSP was determined as the interval from stimulation to the time at which post-stimulus EMG had resumed (Goodall et al. 2010). Provisional data from our laboratory which investigated different contraction strengths, ISIs, and stimulation intensities to produce the greatest SICI response dictated the method used. To elicit SICI, a subthreshold conditioning stimulus $(0.7 \times \mathrm{aMT})$ was followed by a supra-threshold $(1.2 \times \mathrm{aMT})$ unconditioned test stimulus using an inter-stimulus interval (ISI) of $2 \mathrm{~ms}$. The SICI ratio was determined by comparing the amplitude of the conditioned and unconditioned responses. The ratio between the unconditioned MEP and resting $M_{\max }$ was used for the quantification of corticospinal excitability at each respective time point. During all contractions, visual feedback of the target force was provided via a computer monitor.

\section{Data analysis}

Voluntary activation measured through stimulation of the femoral nerve, was quantified using the twitch interpolation technique (Merton 1954). Voluntary activation was quantified by comparing the amplitude of the SIT during MVCs with the amplitude of the resting $Q_{\mathrm{tw}, \text { pot }}$ elicited $2 \mathrm{~s}$ post-MVC: VA $(\%)=\left(1-\left[\mathrm{SIT} / Q_{\mathrm{tw}, \text { pot }}\right] \times 100\right)$. The peak-topeak amplitudes of evoked MEPs and $M_{\max }$ were calculated offline.

\section{Statistical analysis}

Data are presented as means $\pm \mathrm{SD}$ throughout. A one-way repeated measures analysis of variance (ANOVA) was used to assess changes in all outcome measures. Assumptions of sphericity were explored and controlled for all variables using the Greenhouse-Geisser adjustment, where appropriate. Where significant main effects were detected, and pairwise comparisons between time-points are reported, the Tukey method was used to make adjustments for multiple comparisons (Graphpad Prism, v5.04, La Jolla, CA, USA). Statistical significance was assumed at $P \leq 0.05$.

\section{Results}

\section{Neuromuscular function}

Force reduced throughout the protocol $\left(F_{3,36}=52.83\right.$, $P<0.0001$; Fig. 2), with peak values during MVC1 $(-10 \pm 7 \%, P<0.01)$, MVC2 $(-24 \pm 9 \%, P<0.001)$ and MVC3 $(-29 \pm 19 \%, P<0.001)$ decreased compared to baseline $(706 \pm 115 \mathrm{~N})$. The reductions in maximal force during MVC2 and MVC3 were greater than MVC1 $(P<0.001)$ (Fig. 3A). The reduced MVC was accompanied by a significant reduction in $Q_{\mathrm{tw}, \text { pot }}\left(F_{3,36}=135.60\right.$, $P<0.0001$ ) indicative of peripheral fatigue (Fig. 3B). The 


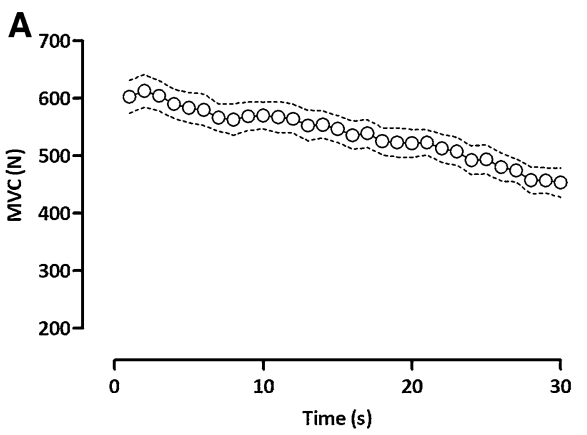

B

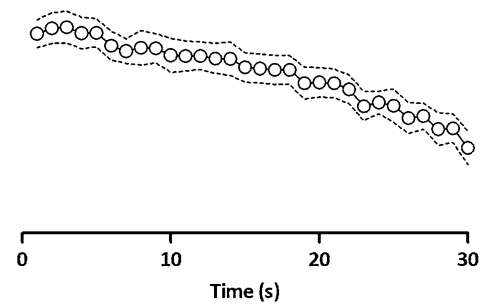

C

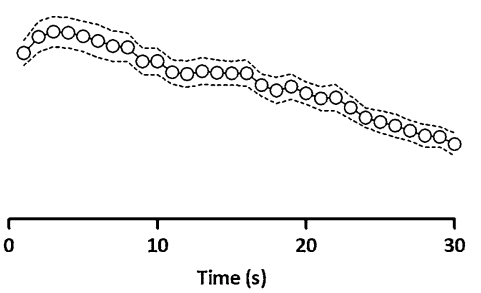

Fig. 2 Average (1 s) knee-extensor force throughout the three sustained, maximal contractions (A MVC1; B MVC2; C MVC3). Values are means \pm SD (dashed lines)

decline in $Q_{\mathrm{tw}, \mathrm{pot}}$ amplitude from baseline $(207 \pm 30 \mathrm{~N})$ was evident following MVC1 $(-23 \pm 10 \%, P<0.001)$ with further reductions following MVC2 $(-53 \pm 13 \%$, $P<0.001)$ and MVC3 $(-60 \pm 13 \%, P<0.001)$. The reductions in $Q_{\mathrm{tw}, \text { pot }}$ following MVC2 and MVC3 were greater than MVC1 $(P<0.001)$. Central fatigue was evident via reductions in VA throughout the protocol $\left(F_{3,36}=9.14\right.$, $P<0.0001)$. In comparison to baseline $(94 \pm 4 \%)$, VA was reduced following MVC2 $(-10 \pm 13 \%, P<0.01)$ and MVC3 $(-13 \pm 10 \%, P<0.001)$. The reduction in VA during MVC2 and MVC3 was greater than MVC1 $(P<0.05$; Fig. 3C).

\section{Corticospinal excitability, CSP and SICI}

There were no changes in $M_{\max }$ amplitude for any kneeextensor muscle $\left(F_{3,36} \leq 0.60, P \geq 0.619\right.$; Fig. 4A); however, MEP amplitudes were reduced throughout the protocol $\left(F_{3,36} \geq 2.89, P \leq 0.015\right)$. Consequently, corticospinal excitability $\left(\mathrm{MEP} / M_{\max }\right)$ was reduced in the knee-extensors following MVC1 (Fig. 4B). CSP duration was similar at baseline in all knee-extensor muscles $(R F$, $178 \pm 21 \mathrm{~ms} ; V L, 176 \pm 25 \mathrm{~ms} ; V M, 166 \pm 25 \mathrm{~ms})$ and was significantly lengthened following MVC1 $(R F, 34 \pm 14 \%$; $\left.V L, 25 \pm 21 \% ; V M, 32 \pm 18 \% ; F_{3,36} \geq 20.90, P<0.001\right)$. CSP duration progressively increased following MVC3 in the $V M(P=0.022$ vs. MVC1; $P=0.050$ vs. MVC2 $)$ and $V L(P=0.015$ vs. MVC1) (Fig. 4C). Baseline SICI was similar in all knee-extensor muscles $(R F, 0.75 \pm 0.17$; $V L, 0.70 \pm 0.17 ; V M, 0.70 \pm 0.18)$ and did not change at any point during the protocol $\left(F_{3,36} \leq 1.34, P \geq 0.276\right.$; Fig. 4D). The changes in MEP amplitude and CSP duration are shown for a representative participant in Fig. 5A, $\mathrm{B}$, respectively. During each assessment period, there were time effects for the unconditioned $R F, V L$ and $V M$ MEP amplitudes and the conditioned $V L$ and $V M$ MEP amplitudes; these responses are shown in Supplementary Fig. 1.

\section{rmsEMG activity}

The rmsEMG $/ M_{\max }$ was reduced in the $V L\left(F_{5,31}=3.94\right.$, $P=0.004)$ during the sustained MVCs, but no changes were observed in the $R F\left(F_{2,28}=2.72, P=0.075\right)$ or $V M$ $\left(F_{2,23}=3.02, P=0.072\right)$ (Supplementary Fig. 2$)$. The prestimulus rmsEMG $/ M_{\max }$ during the assessment sets between sustained contractions was similar for all knee-extensor muscles and did not change after any sustained MVC $(P \geq 0.119)$. Furthermore, there were no time effects for the pre-stimulus rmsEMG measured during the 60 -s assessment periods between each sustained contraction (Supplementary Fig. 3).

\section{Discussion}

This study determined whether the development of fatigue following repeated, isometric, maximal contractions performed with the knee-extensors, altered corticospinal excitability and inhibition. The novel findings of this study are that (1) the fatigue elicited was concurrent with a reduced corticospinal excitability and increased CSP duration, whereas (2) SICI did not change from baseline at any point throughout the protocol. These data demonstrate that the fatigue elicited by repeated, sustained contractions, caused impairments in neuromuscular function coupled with differential responses in excitability and inhibitory networks within the motor cortex. Specifically, activity of inhibitory networks mediated by $\mathrm{GABA}_{\mathrm{B}}$ were increased with fatigue, however, those mediated by $\mathrm{GABA}_{\mathrm{A}}$ were not.

\section{Mechanisms of neuromuscular fatigue}

The sustained maximal contractions elicited progressive neuromuscular fatigue that was accompanied by reductions in maximal force generating capacity (Fig. 3A), resting potentiated twitch force (Fig. 3B) and voluntary activation 

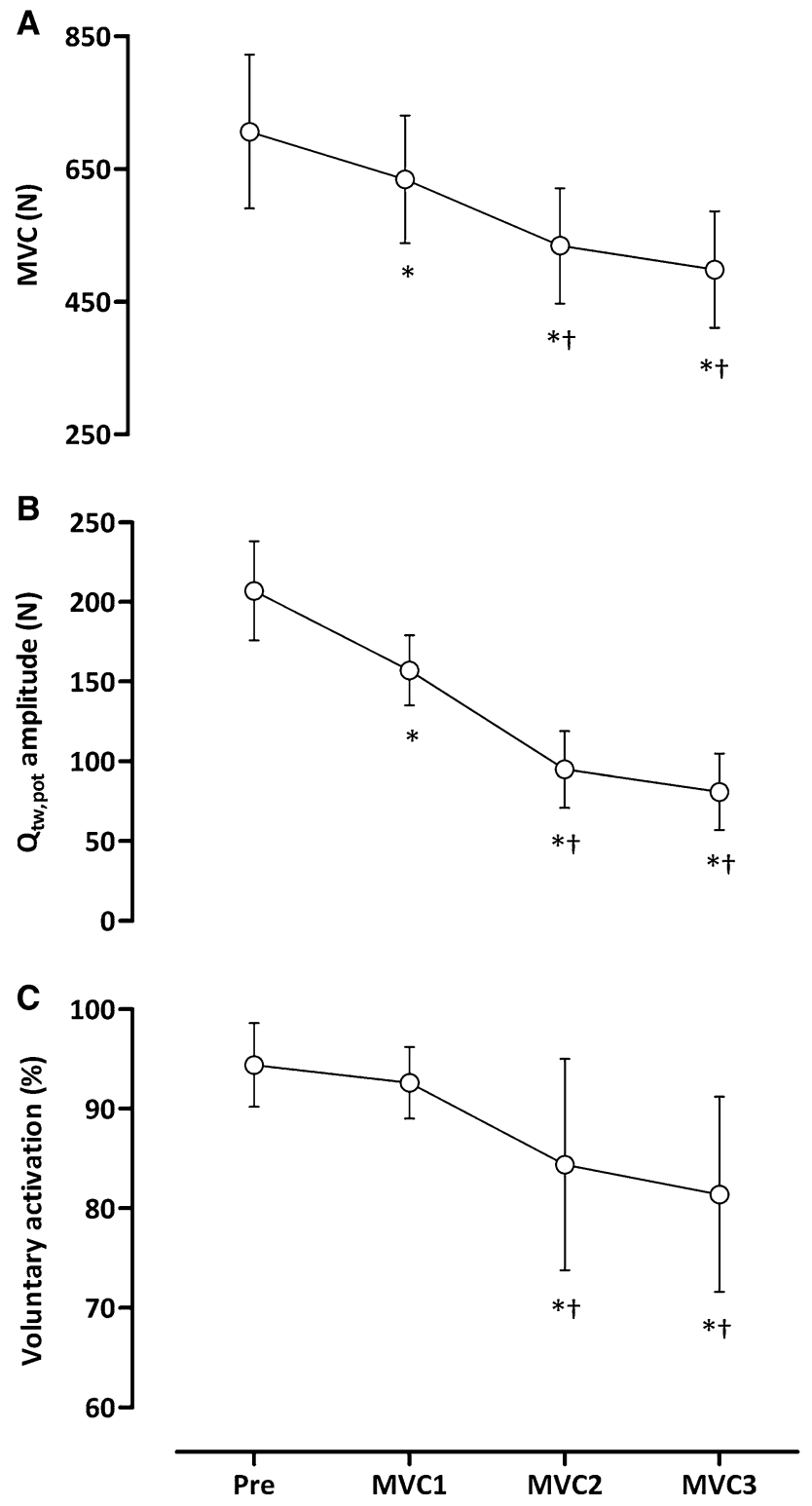

Fig. 3 Maximum voluntary contraction (A), potentiated knee-extensor twitch force $(\mathbf{B})$ and voluntary activation measured with motor nerve stimulation $(\mathbf{C})$ at baseline, and following three sustained, maximal contractions. ${ }^{*} P<0.05$ vs. baseline, ${ }^{\dagger} P<0.05$ vs. MVC1. Values are means $\pm \mathrm{SD}$

(Fig. 3C). The final force attained in the third MVC was reduced by $\sim 40 \%$ compared to baseline; a value that is less than other investigations studying responses in the knee-extensors following a 2-min sustained contraction, where a reduction of $\sim 75 \%$ was observed (Goodall et al. 2009; Place et al. 2009; Kennedy et al. 2016; Vernillo et al. 2017). This is likely because of the shorter total contraction time in the present study, coupled with the minute intervening contractions at $10 \% \mathrm{MVC}$, which would have allowed for greater restoration of blood flow than a sustained 2-min contraction. A restoration of blood flow enables the recovery of muscle force following exerciseinduced fatigue (Kennedy et al. 2016), and might explain why a higher force was observed at the start of each contraction in comparison to the value at the end of the preceding contraction (Fig. 2). The reductions in MVC were accompanied by progressive declines in the $Q_{\mathrm{tw}, \text { pot }}$, indicative of peripheral muscle fatigue (Fig. 3B). The decline in the $Q_{\text {tw.pot }}$ was greatest following MVC3 $(-60 \%)$, which is similar to that observed after sustained (Vernillo et al. 2017) and dynamic (Rossman et al. 2012) knee-extensor exercise, but is more than what is observed following fatiguing locomotor exercise (Amann et al. 2007; Sidhu et al. 2009; Goodall et al. 2012, 2017; Thomas et al. 2016b; Ansdell et al. 2017). As with most investigations that demonstrate peripheral fatigue (Goodall et al. 2010; Rossman et al. 2012; Temesi et al. 2015; Thomas et al. 2016b), the maximal M-wave elicited by supramaximal femoral nerve stimulation was unchanged throughout the experiment. Thus, the peripheral fatigue elicited was likely related to postsynaptic disturbances in the excitation-contraction coupling process. Specifically, impairments to intracellular $\mathrm{Ca}^{+2}$ regulation in the sarcoplasmic reticulum might reduce $\mathrm{Ca}^{+2}$ sensitivity, leading to a reduction in mechanical output and therefore, muscle fatigue (MacIntosh et al. 2012).

There was also a contribution from central mechanisms of fatigue as evidenced by the reduced voluntary activation from MVC2 (Fig. 3C), that is, despite maximal effort, there was insufficient drive to motor units to generate maximal force (Gandevia 2001). During a 2-min maximal contraction of the biceps, Schillings et al. (2003) suggested that mechanisms of peripheral and central fatigue do not change in parallel. Similar to the present study, after approximately $1 \mathrm{~min}$, the contribution of peripheral fatigue tended to level off, and the further decrease in voluntary force can be attributed to mechanisms of central fatigue (Schillings et al. 2003). During the initial part of a sustained contraction the output of muscle is highest, combined with a high metabolic demand and occluded blood flow. Throughout the second half of the contraction, the continuous and repetitive firing of motoneurones would be difficult to maintain, ultimately leading to further decrements in force production (Schillings et al. 2003, 2005; Taylor and Gandevia 2008). Although not measured in the present study, it is likely that that supraspinal mechanisms of fatigue would have contributed to the force loss as the contractions progressed (Gruet et al. 2014). Collectively, these results demonstrate that peripheral fatigue manifests early during the repeated, maximal, sustained contractions; however, with time, there are greater central contributions to the exercise-induced fatigue. 

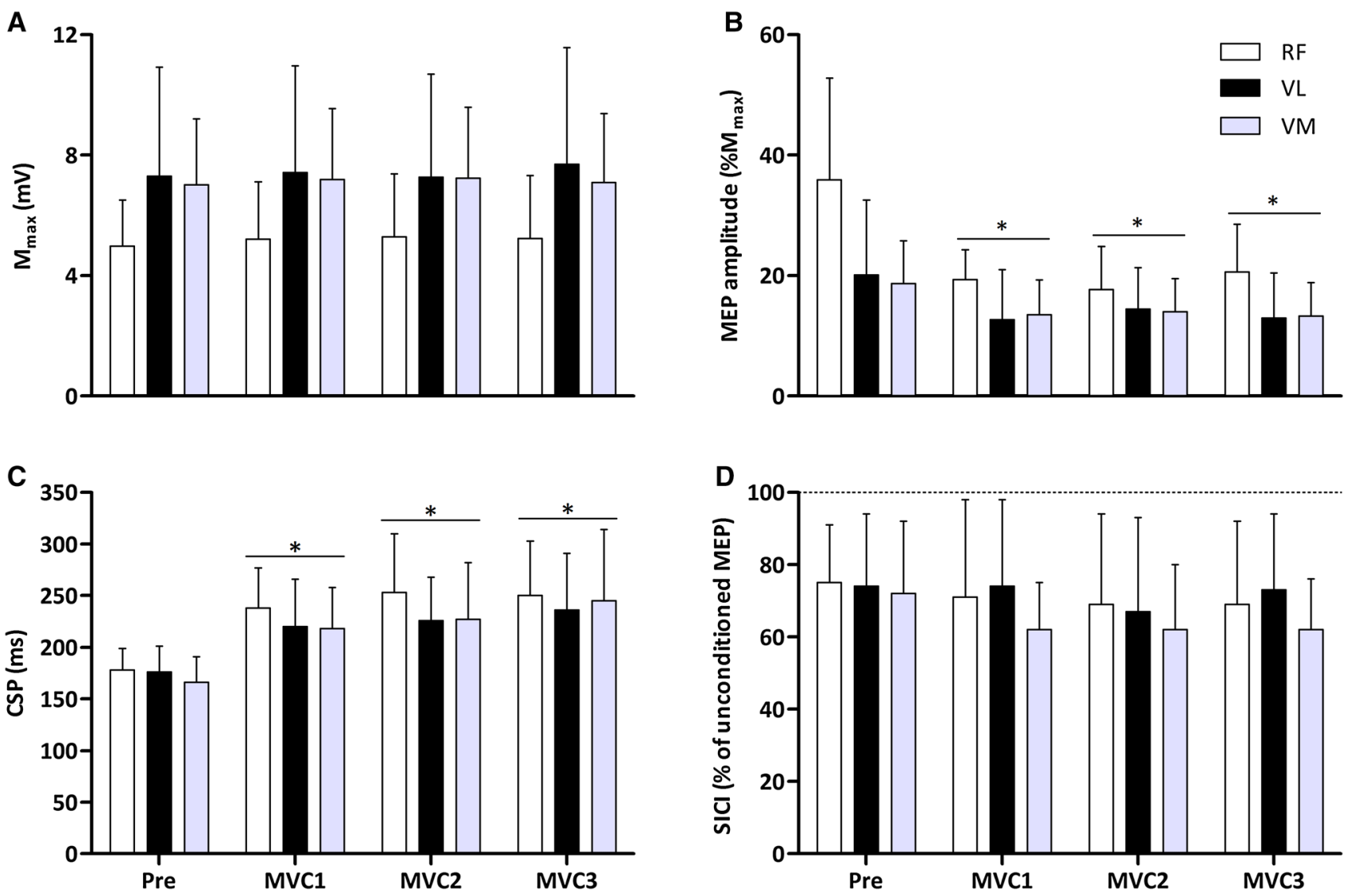

Fig. 4 The maximal M-wave $\left(M_{\max } ; \mathbf{A}\right)$, MEP amplitude $\left(\% M_{\max } ; \mathbf{B}\right)$, the corticospinal silent period (CSP; C) and short-interval intracortical inhibition (SICI) determined during a 10\% MVC contraction (D)

\section{Neuromuscular fatigue and corticospinal excitability}

Since fatigue was observed, the present study was able to investigate the associated changes in corticospinal function with fatigue. Following MVC1, corticospinal excitability was reduced (Fig. 4A) and despite progressive neuromuscular fatigue evident following MVC2 and MVC3, further reductions in corticospinal excitability were not observed. The reduction in corticospinal excitability is opposite to what has recently been reported for the knee-extensors (Kennedy et al. 2016; Vernillo et al. 2017), yet these data are unsurprising because both investigations elicited the MEPs during a sustained contraction. Indeed, this increase in MEP during the fatiguing contraction suggests an increase in the excitability of motor cortical cells (McKay et al. 1996; Taylor et al. 1996; Hilty et al. 2011); however, shortly after termination of a sustained contraction, MEPs either return to baseline (Kennedy et al. 2016) or reduce (Kotan et al. 2015). Some of the proposed mechanisms for the depression in corticospinal excitability include changes in membrane properties of corticospinal neurons and changes in the efficiency at baseline, and following three sustained, maximal knee-extensor contractions. ${ }^{*} P<0.05$ vs. baseline. Values are means $\pm \mathrm{SD}$

of excitatory synaptic inputs onto corticospinal neurons that occur as a result of high levels of activity during the fatiguing contraction (Brasil-Neto et al. 1993; McKay et al. 1996; Samii et al. 1996; Taylor et al. 1996; Kotan et al. 2015). A change in MEP size, however, does not solely reflect changes at the motor cortex, as these potentials can also be affected by changes in the motoneurones and muscle fibres (Taylor and Gandevia 2004; Taylor 2006; McNeil et al. 2009). Furthermore, corticospinal excitability has also been shown to reduce in response to a period of constant electrical stimulation of the median nerve (Kotan et al. 2015), demonstrating a role for afferent feedback. It is well known that Group III and IV muscle afferents fire during continuous muscle contraction in response to mechanical and metabolic changes during skeletal muscle contraction (Taylor and Gandevia 2008), and previous work has shown such afferent feedback to reduce corticospinal excitability (Ridding and Rothwell 1997; Ziemann et al. 1998). Despite these findings, a recent investigation has found excitability of the $V L$ motoneurone pool to be unaffected by feedback from Group III and IV muscle afferents (Kennedy et al. 2016). Nevertheless, high levels of cortical activity during the sustained contractions, along 
Fig. 5 A shows the MEP response in each knee-extensor muscle, the horizontal dashed line demonstrates the reduction in amplitude in comparison to baseline, following the 3rd sustained MVC. B shows the corticospinal silent period measured in each knee-extensor muscle (see "Methods" for quantification). The vertical dashed lines are placed at the time of stimulation and at the end of the silent period, the traces on the right show increased silent period durations following the 3rd sustained MVC. For each panel, respectively, data are from a representative participant and the responses to six unconditioned motor cortex stimuli are overlaid. $R F$ rectus femoris, $V L$ vastus lateralis, $V M$ vastus medialis
A

Baseline

Post MVC3

B Baseline

Post MVC3
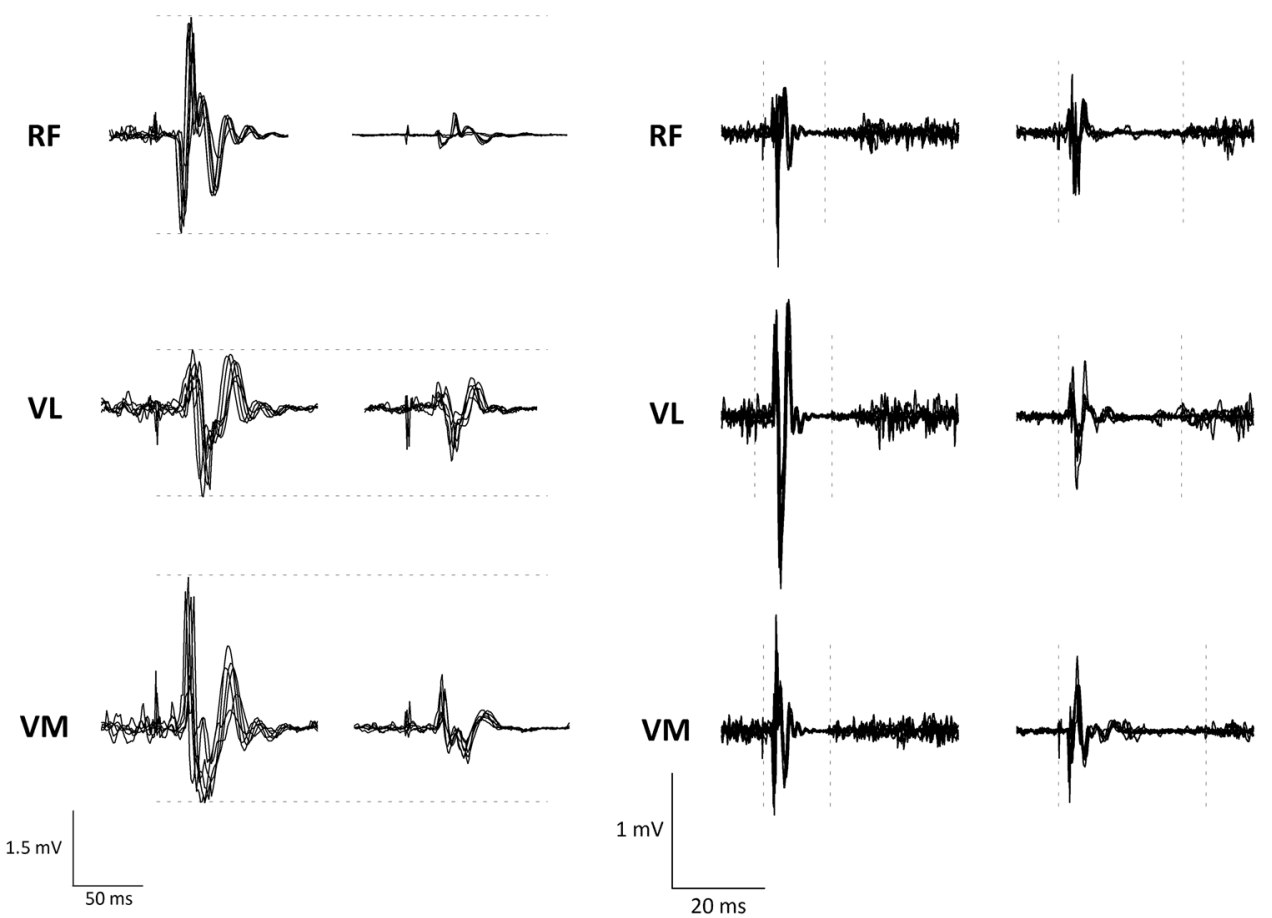

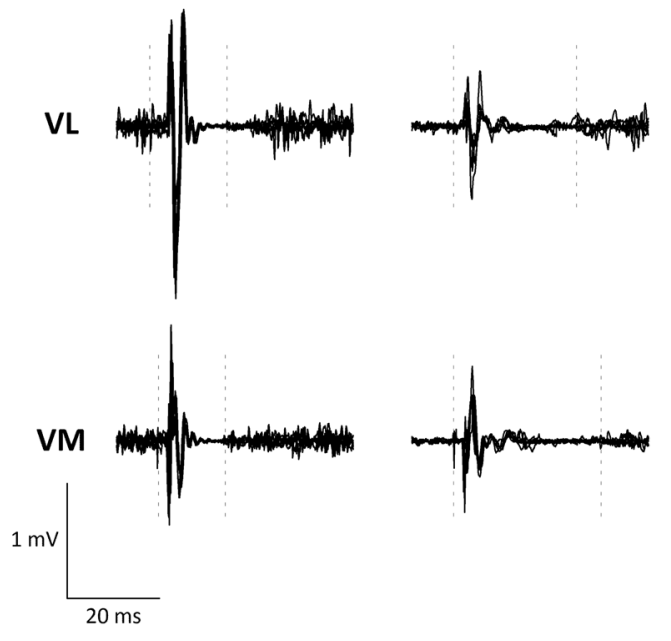

with a heightened afferent feedback, are plausible explanations for the reduced corticospinal excitability observed in the present study.

\section{Neuromuscular fatigue and motor cortical inhibition}

The diminished corticospinal excitability in all kneeextensor muscles occurred in parallel with increased inhibition, in the form of lengthened silent periods (Figs. 4B, 5B). CSP duration was increased compared to baseline in all knee-extensor muscles following all MVCs. As the protocol ensued, progressive increases in CSP duration were observed ( $V M$ and $V L)$ signifying an augmented level of inhibition. For many years it has been thought that spinal mechanisms contribute to the initial (50-80 ms) part of the CSP (Fuhr et al. 1991; Ziemann et al. 1993). However, more recent evidence suggests that the CSP has a much longer, and more influential spinal component than previously thought (Yacyshyn et al. 2016). The lengthening of CSP duration when fatigue is experienced, has been attributed to increased cortical inhibition only (Kennedy et al. 2016). Kennedy et al. (2016) have recently shown unchanged motoneuronal responsiveness, but increased CSP duration, following fatiguing isometric exercise performed with the knee-extensors. These data, coupled with the present study, suggest that fatigue-related afferents influence inhibition mediated by $\mathrm{GABA}_{\mathrm{B}}$ receptors within the motor cortex.
These data confirm previous observations from blockade studies showing that when feedback from such afferents is blocked, by the injection of intrathecal fentanyl, CSP duration does not increase (Hilty et al. 2011; Sidhu et al. 2017). Conversely, SICI, which tests the excitability of $\mathrm{GABA}_{\mathrm{A}}$-mediated intracortical inhibitory networks within the motor cortex was unaffected by fatigue. At baseline, SICI measured in the active knee-extensors, was similar to that previously reported ( 0.75; Brownstein et al. 2017; Thomas et al. 2017b). However, in contrast to the reduction in corticospinal excitability following MVC1, SICI was unaltered following any of the sustained contractions (Fig. 4C); which concurs with some literature (Tergau et al. 2000; Brownstein et al. 2017; Thomas et al. 2017a), but not others, who report reduced inhibition (Benwell et al. 2006; Maruyama et al. 2006; Takahashi et al. 2011; Hunter et al. 2016).

Our data suggest that $\mathrm{GABA}_{\mathrm{B}}$ receptors play an important role in modulating the intracortical inhibitory response within the motor cortex during fatiguing exercise. The effect of GABA released by the presynaptic axon terminals of inhibitory interneurons depends on the receptor on the postsynaptic cell membrane it interacts with. McCormick (1992) explained how $\mathrm{GABA}_{\mathrm{A}}$ receptors are mediated by a short-lasting, $\mathrm{Cl}^{-}$-dependant, component of stimulationinduced inhibitory postsynaptic potentials; whereas, a longlasting $\mathrm{K}^{+}$-dependent component, results from activation of postsynaptic $\mathrm{GABA}_{\mathrm{B}}$ receptors. The latter component is only evident when inhibitory interneurons are strongly 
activated; suggesting that activation of $\mathrm{GABA}_{\mathrm{B}}$ receptors requires a higher GABA concentration or a longer exposure to GABA than that necessary for the activation of $\mathrm{GABA}_{\mathrm{A}}$ receptors (Otis and Mody 1992). Thus, the fatigue elicited by the repeated, sustained contractions, was likely in parallel with a substantial release of GABA from inhibitory interneurons. Such a release of GABA is not easily resolved in the synaptic cleft, thus favouring $\mathrm{GABA}_{\mathrm{B}}$ receptor activation (Thompson and Gahwiler 1992), explaining the prolonged CSP but unchanged SICI.

The conflicting results within the literature for the response in SICI during fatigue are possibly due to subtle differences in the way in which SICI is assessed. For example, SICI will depend on whether the muscle is in an active or resting state, whether the unconditioned MEP size is of a specific amplitude rather than being based on $1.2 \times \mathrm{aMT}$, by varying the strength of sub-threshold conditioning stimulations, and altering the inter-stimulus interval. Many of these variables can be manipulated (Ortu et al. 2008) and it is important to optimise the methods of assessing SICI in a particular muscle prior to studying a response. Provisional data from our laboratory which investigated different contraction strengths, ISIs, and stimulation intensities to produce the greatest SICI response dictated the method used in the present study. Rather than basing the test pulse on a specific MEP size, we use $1.2 \times \mathrm{aMT}$, with the aMT determined during a $10 \%$ MVC. Indeed, with changes in corticospinal excitability following MVC1, thresholds may have also changed. However, it was not possible to adjust the stimulator output to achieve a specific MEP size, or to re-establish thresholds during the 60 -s assessment period between the sustained contractions. Rather, we elicited MEP and SICI measurements with a consistent level of mechanical output (relative 10\% MVC) at all-time points. It was important that we controlled for the level of mechanical output because assessing SICI at the absolute 10\% MVC level would mean the contraction strength would have been higher when the muscle was in a fatigued state. Indeed, the minute between the sustained contractions would had presumably allowed for some recovery in force, such that, towards the end of the 60-s period the contraction held might have been more akin to around 5, rather than $10 \%$ MVC. The time effects observed for MEP and SICI amplitudes throughout these assessment periods can be explained by the first 1 or 2 responses following each sustained MVC, being larger than the following 4 or 5 responses (see Supplementary Fig. 1). There were no changes in the $M_{\max }$ at these time points and the larger MEP responses immediately post the sustained MVCs are not deemed to be 'facilitated', because they are no more than the baseline value. Similar findings, elicited in resting muscle, have previously been reported and termed central postactivation facilitation, which is related to the balance of neurotransmitters during fatigue (Brasil-Neto et al. 1993, 1994). For these increased, isolated responses, a greater level of inhibition is observed (reduced SICI ratio); however, this was not representative of the mean response during each assessment period (Fig. 4D). Thus, we do not believe this finding changes our conclusions as the responses to TMS should not be observed in isolation, it is far more prudent to study the mean of a set of responses as is done in the present study. Moreover, the evoked response during each assessment period were elicited with the same level of pre-stimulus EMG (Supplementary Fig. 3), thus, we do not believe the effect of 'recovery' throughout the 60-s assessment period impacts the conclusions made in the present study.

\section{Conclusion}

In summary, our findings indicate that a progressive level of neuromuscular fatigue within the knee-extensors coincides with a reduced corticospinal excitability and increased CSP duration, demonstrating modulation of specific neural networks within the corticospinal pathway. Additionally, our data reveal changes in the behaviour of networks within the motor cortex with fatigue and future work should aim to understand the mechanisms for the differential responses in inhibitory networks mediated by GABA receptors.

Acknowledgements We thank Sam Bacon, Andrew Martins and Alan Toward for the assistance with data collection and processing.

Open Access This article is distributed under the terms of the Creative Commons Attribution 4.0 International License (http://creativecommons.org/licenses/by/4.0/), which permits unrestricted use, distribution, and reproduction in any medium, provided you give appropriate credit to the original author(s) and the source, provide a link to the Creative Commons license, and indicate if changes were made.

\section{References}

Amann M, Romer LM, Subudhi AW, Pegelow DF, Dempsey JA (2007) Severity of arterial hypoxaemia affects the relative contributions of peripheral muscle fatigue to exercise performance in healthy humans. J Physiol 581:389-403

Ansdell P, Thomas K, Howatson G, Amann M, Goodall S (2017) Deception improves TT performance in well-trained cyclists without augmented fatigue. Med Sci Sports Exerc. https://doi. org/10.1249/MSS.0000000000001483

Benwell NM, Sacco P, Hammond GR, Byrnes ML, Mastaglia FL, Thickbroom GW (2006) Short-interval cortical inhibition and corticomotor excitability with fatiguing hand exercise: a central adaptation to fatigue? Exp Brain Res 170:191-198

Brasil-Neto JP, Pascual-Leone A, Valls-Sole J, Cammarota A, Cohen LG, Hallett M (1993) Postexercise depression of motor evoked potentials: a measure of central nervous system fatigue. Exp Brain Res 93:181-184 
Brasil-Neto JP, Cohen LG, Hallett M (1994) Central fatigue as revealed by postexercise decrement of motor evoked potentials. Muscle Nerve 17:713-719

Brownstein CG, Dent JP, Parker P, Hicks KM, Howatson G, Goodall S, Thomas K (2017) Etiology and recovery of neuromuscular fatigue following competitive soccer match-play. Front Physiol. https:// doi.org/10.3389/fphys.2017.00831

Carroll TJ, Taylor JL, Gandevia SC (2017) Recovery of central and peripheral neuromuscular fatigue after exercise. J Appl Physiol 122:1068-1076

Chen R, Lozano AM, Ashby P (1999) Mechanism of the silent period following transcranial magnetic stimulation. Evidence from epidural recordings. Exp Brain Res 128:539-542

Di Lazzaro V, Oliviero A, Meglio M, Cioni B, Tamburrini G, Tonali P, Rothwell JC (2000) Direct demonstration of the effect of lorazepam on the excitability of the human motor cortex. Clin Neurophysiol 111:794-799

Fuhr P, Agostino R, Hallett M (1991) Spinal motor neuron excitability during the silent period after cortical stimulation. Electroencephalogr Clin Neurophysiol 81:257-262

Gandevia SC (2001) Spinal and supraspinal factors in human muscle fatigue. Physiol Rev 81:1725-1789

Goodall S, Romer LM, Ross EZ (2009) Voluntary activation of human knee extensors measured using transcranial magnetic stimulation. Exp Physiol 94:995-1004

Goodall S, Ross EZ, Romer LM (2010) Effect of graded hypoxia on supraspinal contributions to fatigue with unilateral kneeextensor contractions. J Appl Physiol 109:1842-1851

Goodall S, Gonzalez-Alonso J, Ali L, Ross EZ, Romer LM (2012) Supraspinal fatigue after normoxic and hypoxic exercise in humans. J Physiol 590:2767-2782

Goodall S, Charlton K, Hignett C, Prichard J, Barwood M, Howatson G, Thomas K (2015) Augmented supraspinal fatigue following constant-load cycling in the heat. Scand J Med Sci Sports 25(Suppl 1):164-172

Goodall S, Thomas K, Harper LD, Hunter R, Parker P, Stevenson E, West D, Russell M, Howatson G (2017) The assessment of neuromuscular fatigue during $120 \mathrm{~min}$ of simulated soccer exercise. Eur J Appl Physiol 117:687-697

Gruet M, Temesi J, Rupp T, Levy P, Millet GY, Verges S (2013) Stimulation of the motor cortex and corticospinal tract to assess human muscle fatigue. Neuroscience 231:384-399

Gruet M, Temesi J, Rupp T, Levy P, Verges S, Millet GY (2014) Dynamics of corticospinal changes during and after high-intensity quadriceps exercise. Exp Physiol 99:1053-1064

Hilty L, Lutz K, Maurer K, Rodenkirch T, Spengler CM, Boutellier U, Jancke L, Amann M (2011) Spinal opioid receptor-sensitive muscle afferents contribute to the fatigue-induced increase in intracortical inhibition in healthy humans. Exp Physiol 96:505-517

Hunter SK, McNeil CJ, Butler JE, Gandevia SC, Taylor JL (2016) Short-interval cortical inhibition and intracortical facilitation during submaximal voluntary contractions changes with fatigue. Exp Brain Res 234:2541-2551

Kennedy DS, McNeil CJ, Gandevia SC, Taylor JL (2016) Effects of fatigue on corticospinal excitability of the human knee extensors. Exp Physiol 101:1552-1564

Kotan S, Kojima S, Miyaguchi S, Sugawara K, Onishi H (2015) Depression of corticomotor excitability after muscle fatigue induced by electrical stimulation and voluntary contraction. Front Hum Neurosci 9:363

Kujirai T, Caramia MD, Rothwell JC, Day BL, Thompson PD, Ferbert A, Wroe S, Asselman P, Marsden CD (1993) Corticocortical inhibition in human motor cortex. J Physiol 471:501-519
Lentz M, Nielsen JF (2002) Post-exercise facilitation and depression of $\mathrm{M}$ wave and motor evoked potentials in healthy subjects. Clin Neurophys 113:1092-1098

MacIntosh BR, Holash RJ, Renaud JM (2012) Skeletal muscle fatigue-regulation of excitation-contraction coupling to avoid metabolic catastrophe. J Cell Sci 125:2105-2114

Maruyama A, Matsunaga K, Tanaka N, Rothwell JC (2006) Muscle fatigue decreases short-interval intracortical inhibition after exhaustive intermittent tasks. Clin Neurophysiol 117:864-870

McCormick DA (1992) Neurotransmitter actions in the thalamus and cerebral cortex. J Clin Neurophysiol 9:212-223

McDonnell MN, Orekhov Y, Ziemann U (2006) The role of GABA(B) receptors in intracortical inhibition in the human motor cortex. Exp Brain Res 173:86-93

McKay WB, Stokic DS, Sherwood AM, Vrbova G, Dimitrijevic MR (1996) Effect of fatiguing maximal voluntary contraction on excitatory and inhibitory responses elicited by transcranial magnetic motor cortex stimulation. Muscle Nerve 19:1017-1024

McNeil CJ, Martin PG, Gandevia SC, Taylor JL (2009) The response to paired motor cortical stimuli is abolished at a spinal level during human muscle fatigue. J Physiol 587:5601-5612

Merton PA (1954) Voluntary strength and fatigue. J Physiol 123:553-564

Ni Z, Chen R (2011) Excitatory and Inhibitory Effects of Transcranial Magnetic Stimulation. Biocyber Biomed Eng 31:93-105

Ortu E, Deriu F, Suppa A, Tolu E, Rothwell JC (2008) Effects of volitional contraction on intracortical inhibition and facilitation in the human motor cortex. J Physiol 586:5147-5159

Otis TS, Mody I (1992) Differential activation of GABAA and GABAB receptors by spontaneously released transmitter. J Neurophysiol 67:227-235

Place N, Bruton JD, Westerblad H (2009) Mechanisms of fatigue induced by isometric contractions in exercising humans and in mouse isolated single muscle fibres. Clin Exp Pharmacol Physiol 36:334-339

Ridding MC, Rothwell JC (1997) Stimulus/response curves as a method of measuring motor cortical excitability in man. Electroencephalogr Clin Neurophysiol 105:340-344

Rossman MJ, Venturelli M, McDaniel J, Amann M, Richardson RS (2012) Muscle mass and peripheral fatigue: a potential role for afferent feedback? Acta Physiol 206:242-250

Rothwell JC (2009) The fatigued spinal cord. J Physiol 587:5517-5518

Samii A, Wassermann EM, Ikoma K, Mercuri B, Hallett M (1996) Characterization of postexercise facilitation and depression of motor evoked potentials to transcranial magnetic stimulation. Neurology 46:1376-1382

Schillings ML, Hoefsloot W, Stegeman DF, Zwarts MJ (2003) Relative contributions of central and peripheral factors to fatigue during a maximal sustained effort. Eur J Appl Physiol 90:562-568

Schillings ML, Stegeman DF, Zwarts MJ (2005) Determining central activation failure and peripheral fatigue in the course of sustained maximal voluntary contractions: a model-based approach. J Appl Physiol 98:2292-2297

Sidhu SK, Bentley DJ, Carroll TJ (2009) Locomotor exercise induces long-lasting impairments in the capacity of the human motor cortex to voluntarily activate knee extensor muscles. J Appl Physiol 106:556-565

Sidhu SK, Weavil JC, Mangum TS, Jessop JE, Richardson RS, Morgan DE, Amann M (2017) Group III/IV locomotor muscle afferents alter motor cortical and corticospinal excitability and promote central fatigue during cycling exercise. Clin Neurophysiol 128:44-55

Takahashi K, Maruyama A, Maeda M, Etoh S, Hirakoba K, Kawahira K, Rothwell JC (2009) Unilateral grip fatigue reduces short 
interval intracortical inhibition in ipsilateral primary motor cortex. Clin Neurophysiol 120:198-203

Takahashi K, Maruyama A, Hirakoba K, Maeda M, Etoh S, Kawahira K, Rothwell JC (2011) Fatiguing intermittent lower limb exercise influences corticospinal and corticocortical excitability in the nonexercised upper limb. Brain Stimul 4:90-96

Taylor JL (2006) Stimulation at the cervicomedullary junction in human subjects. J Electromyogr Kinesiol 16:215-223

Taylor JL, Gandevia SC (2001) Transcranial magnetic stimulation and human muscle fatigue. Muscle Nerve 24:18-29

Taylor JL, Gandevia SC (2004) Noninvasive stimulation of the human corticospinal tract. J Appl Physiol 96:1496-1503

Taylor JL, Gandevia SC (2008) A comparison of central aspects of fatigue in submaximal and maximal voluntary contractions. $\mathrm{J}$ Appl Physiol 104:542-550

Taylor JL, Butler JE, Allen GM, Gandevia SC (1996) Changes in motor cortical excitability during human muscle fatigue. J Physiol 490:519-528

Temesi J, Arnal PJ, Rupp T, Feasson L, Cartier R, Gergele L, Verges S, Martin V, Millet GY (2015) Are Females More Resistant to Extreme Neuromuscular Fatigue? Med Sci Sports Exerc 47:1372-1382

Tergau F, Geese R, Bauer A, Baur S, Paulus W, Reimers CD (2000) Motor cortex fatigue in sports measured by transcranial magnetic double stimulation. Med Sci Sports Exerc 32:1942-1948

Thomas AC, Pietrosimone BG, Bayer CJ (2016a) Agreement between investigators using paired-pulse transcranial magnetic stimulation to assess quadriceps intracortical excitability. J Sport Rehabil: $1-13$

Thomas K, Elmeua M, Howatson G, Goodall S (2016b) Intensitydependent contribution of neuromuscular fatigue after constantload cycling. Med Sci Sports Exerc 48:1751-1760

Thomas K, Dent J, Howatson G, Goodall S (2017a) Etiology and recovery of neuromuscular fatigue after simulated soccer match play. Med Sci Sports Exerc 49:955-964
Thomas K, Toward A, West DJ, Howatson G, Goodall S (2017b) Heavy-resistance exercise-induced increases in jump performance are not explained by changes in neuromuscular function. Scand $\mathbf{J}$ Med Sci Sports 27:35-44

Thompson SM, Gahwiler BH (1992) Effects of the GABA uptake inhibitor tiagabine on inhibitory synaptic potentials in rat hippocampal slice cultures. J Neurophysiol 67:1698-1701

Vernillo G, Temesi J, Martin M, Millet GY (2017) Mechanisms of fatigue and recovery in upper versus lower limbs in men. Med Sci Sports Exerc. https://doi.org/10.1249/MSS.0000000000001445

Williams PS, Hoffman RL, Clark BC (2014) Cortical and spinal mechanisms of task failure of sustained submaximal fatiguing contractions. PLoS One 9:e93284

Yacyshyn AF, Woo EJ, Price MC, McNeil CJ (2016) Motoneuron responsiveness to corticospinal tract stimulation during the silent period induced by transcranial magnetic stimulation. Exp Brain Res 234:3457-3463

Yoon T, Schlinder-Delap B, Keller ML, Hunter SK (2012) Supraspinal fatigue impedes recovery from a low-intensity sustained contraction in old adults. J Appl Physiol 112:849-858

Ziemann U, Netz J, Szelenyi A, Homberg V (1993) Spinal and supraspinal mechanisms contribute to the silent period in the contracting soleus muscle after transcranial magnetic stimulation of human motor cortex. Neurosci Lett 156:167-171

Ziemann U, Lonnecker S, Steinhoff BJ, Paulus W (1996) The effect of lorazepam on the motor cortical excitability in man. Exp Brain Res 109:127-135

Ziemann U, Hallett M, Cohen LG (1998) Mechanisms of deafferentation-induced plasticity in human motor cortex. J Neurosci 18:7000-7007 